\title{
Evidências de Validade da Versão de Heterorrelato do Inventário de Clima Familiar em uma Amostra de Cuidadores de Crianças
}

\author{
Laila Akerman, Juliane Callegaro Borsa ${ }^{1}$ \\ Pontifícia Universidade Católica do Rio de Janeiro, Rio de Janeiro-RJ, Brasil
}

RESUMO

O clima familiar refere-se à percepção de características dos relacionamentos intrafamiliares por seus membros. O presente estudo visa investigar evidências de validade baseada na estrutura interna da versão de heterorrelato do Inventário de Clima Familiar. Participaram do estudo 722 cuidadores (76,3 \% mulheres) de crianças (52,4\% meninas) com idades entre oito e 12 anos $\left(M_{\text {idade }}=9,17 ; D P=1,59\right)$. Análises Fatoriais Exploratórias (AFE) indicaram propriedades psicométricas adequadas (Kaiser-MeyerOlkin $[\mathrm{KMO}]=0,90049 ;$ Bartlett's test of sphericity $=5850,8 ; d f=231 ; p<0,001$; RMSEA=0,058; NNFI=0.,971; WRMR=0,0537) e uma solução de dois fatores da versão de heterorrelato do ICF, sendo eles Clima Familiar Positivo e Clima Familiar Negativo, em consistência com a literatura sobre clima familiar.

Palavras-chave: família; clima familiar; inventário; heterorrelato.

\begin{abstract}
Evidence of validity for the observer-report version of the Family Climate Inventory in a sample of caregivers of children

The family climate refers to the perception of family relationship characteristics by its members. The present study aimed to investigate the evidence of validity of the observer-report version of the Family Climate Inventory based on its internal structure. A total of 722 caregivers $\left(76.3 \%\right.$ women) of children ( $52.4 \%$ girls) aged between 8 and 12 years $\left(M_{\text {age }}=9.17, S D=1.59\right)$ participated in the study. Exploratory factor analysis indicated adequate psychometric properties (Kaiser-Meyer-Olkin $[\mathrm{KMO}]=0.90049 ;$ Bartlett's test of sphericity $=5850.8 ; d f=231 ; p<0.001$; RMSEA $=0.058$; NNFI $=0.971$; WRMR $=0.0537$ ) and a two factor solution for the observerreport version. The factors Positive Family Climate and Negative Family Climate are consistent with the literature on family climate. Keywords: family; family climate; inventory; observer-report.
\end{abstract}

RESUMEN - Evidencias de validez de la versión de heterorrelato de la Escala de Clima Familiar en una muestra de cuidadores de niños

Se considera Clima Familiar a la percepción de características de las relaciones intrafamiliares por sus miembros. El presente estudio buscó evidencias de validez basadas en la estructura interna de la versión de heterorrelato de la Escala de Clima Familiar. Participaron del estudio 722 cuidadores ( $76.3 \%$ mujeres) de niños $\left(52.4 \%\right.$ de sexo femenino) con edades entre 8 y 12 años $\left(M_{\text {edad }}=9.17, D S=1.59\right)$. El Análisis Factorial Exploratorio indicó propiedades psicométricas adecuadas (Kaiser-Meyer-Olkin [KMO]=0.90049; Bartlett's test of sphericity $=5850.8 ; d f=231 ; p<0.001$; RMSEA=0.058; NNFI=0.971; WRMR=0.0537), y también una solución de dos factores de la versión de heterorrelato del ICF, siendo ellos: Clima Familiar Positivo y Clima Familiar Negativo, corroborando con la literatura existente sobre clima familiar

Palabras clave: familia, clima familiar; escala; heterorrelato.

Ao longo da história, a família vem sendo amplamente estudada no campo das ciências humanas e sociais, dado seu papel fundamental para o desenvolvimento social e emocional do indivíduo (Bronfenbrenner, 1996; Minuchin, 1995). Dentre os fatores pertinentes ao contexto familiar está o clima familiar, definido como a percepção dos membros da família sobre os relacionamentos intrafamiliares e sobre as estratégias orientadas ao controle e manutenção do sistema familiar (Moos \& Moos, 1994; Teodoro, Allgayer, \& Land, 2009).
Moos e Moos (1994) destaca a complexidade e a importância de diferentes dimensões na composição do clima familiar, como o grau de envolvimento, apoio, compromisso, suporte e ajuda oferecida pela família e a manifestação de raiva, agressão e conflito entre os membros, objetivos relacionados ao crescimento pessoal para os quais o ambiente familiar é orientado, extensão em que os membros da família são assertivos, independentes, autossuficientes, estratégias utilizadas para a manutenção do sistema familiar, a ordem e a estrutura da família,

${ }^{1}$ Endereço para correspondência: Pontifícia Universidade Católica do Rio de Janeiro, Departamento de Psicologia. Rua Marquês de São Vicente, 225, Prédio Cardeal Leme, Sala 201, 22451-900. E-mail: juliborsa@gmail.com

Este projeto foi financiado pelo CNPq 
a abertura à mudanças e o estabelecimento de regras e procedimentos de controle para a gestão familiar (Moos \& Moos, 1994). Em complemento, para Björnberg e Nicholson (2007), a família é um sistema dinâmico em constante busca de equilíbrio, e cujas partes integrantes e subsistemas são interdependentes. Segundo os autores, a partir dessa compreensão sistêmica, algumas características importantes precisam ser consideradas ao se buscar uma compreensão sobre o clima familiar. A primeira deles refere-se à percepção da família como uma unidade que soluciona problemas. Assim, é importante haver coesão e adaptabilidade frente ao ambiente e a resolução de problemas internos e externos à família.

Outra característica importante associa-se ao clima social e relações interpessoais, incluindo as características dos relacionamentos intrafamiliares, aspectos da aprendizagem social entre os membros e o desenvolvimento de psicopatologias na família, por exemplo. Por fim, outra característica relaciona-se à autoridade, às relações de poder e de controle nas relações intergeracionais dentro da família e às estratégias educativas dentro da família (Björnberg \& Nicholson, 2007). Em linhas gerais, os estudos sobre clima familiar destacam dois polos de características, um negativo e outro positivo. Dentre as características negativas que compõem o clima familiar, está a pouca coesão entre os membros, a percepção de afeto inadequado na família, as dificuldades de comunicação e o autoritarismo. Já o polo positivo, refere-se às características de harmonia, segurança, afeto e apoio (Björnberg \& Nicholson, 2007; Teodoro et al., 2009).

Estudos têm apontado a importância do clima familiar para a saúde mental nos membros e para desenvolvimento interpessoal do indivíduo (Ackerman et al., 2013; Leusin, Petrucci, \& Borsa, 2018; Luebbe \& Bell, 2014). Por exemplo, o polo negativo do clima familiar associa-se a comportamentos agressivos na infância (BrophyHerb et al., 2013; Valdés Cuervo \& Carlos Martínez, 2014; Leusin et al., 2018), maiores níveis de ansiedade e depressão (Luebbe \& Bell, 2014), impulsividade, baixa regulação emocional (Brophy-Herb et al., 2013), dificuldades de concentração (Matos et al., 2014), dificuldades nos relacionamentos interpessoais (Petrucci et al., 2016), problemas de isolamento e queixas somáticas (Leusin et al., 2018). O clima familiar positivo, por sua vez, associa-se a maiores níveis de bem-estar (Phillips, 2012), ao desenvolvimento de relações interpessoais íntimas adaptativas, empatia, comportamentos pró-sociais, desenvolvimento emocional positivo (Ackerman et al., 2013), melhor desempenho acadêmico (Marturano \& Elias, 2016).

Levando em consideração a relevância das relações familiares para o desenvolvimento do indivíduo e considerando a carência de instrumentos brasileiros para avaliar o clima familiar, Teodoro et al. (2009) construíram o Inventário de Clima Familiar (ICF), para a avaliação do clima familiar em adolescentes. Para a construção do ICF, os autores definiram operacionalmente o construto a partir da proposta teórica de Björnberg e Nicholson (2007) e de instrumentos internacionais, como o Family Climate Inventory (Kurdek, Fine, \& Sinclair, 1995), o Family Adaptability and Cohesion Evaluation Scale (Olson, Portner, \& Lavee, 1985) e nas principais concepções descritas na literatura e sintetizadas por Björnberg e Nicholson (2007).

O instrumento é composto por 22 itens para serem respondidos segundo uma escala tipo Likert de cinco pontos de concordância (1 "não concordo de jeito nenhum" a 5 "concordo plenamente"). Os itens compõem quatro dimensões: conflito, hierarquia (polo negativo), apoio e coesão familiar (polo positivo). Quanto ao polo negativo, a hierarquia diz respeito ao poder e controle nas relações intergeracionais, o conflito refere-se à presença de agressividade, crítica e relações conflituosas entre os membros da família. Quanto ao polo positivo, o apoio indica a existência de suporte material e emocional e a coesão refere-se ao vínculo afetivo entre os membros da família (Teodoro et al., 2009).

No referido estudo, os autores realizaram análises fatoriais exploratórias (AFE) e análises fatoriais confirmatórias (AFC) para avaliar a estrutura fatorial do ICF, decidindo pela exclusão de itens com carga fatorial e comunalidade $<0,30$. A partir desses procedimentos, foi encontrada uma solução de quatro fatores, nomeadas pelos autores como "Conflito" (6 itens; $\alpha=0,84$ ), "Hierarquia" (6 itens; $\alpha=0,71$ ), "Apoio" ( 5 itens; $\alpha=0,71$ ) e "Coesão ( 5 itens; $\alpha=0,82$ ). O índice Kaiser-Meyer-Olkin (KMO) foi superior a 0,80 e o teste de esfericidade de Bartlett foi significativo $\left(\chi^{2}=1996,33 ; g l=231, p<0,001\right)$. Todos os fatores do ICF apresentaram correlações significativas entre si. O fator Conflito correlacionou-se positivamente com o fator Hierarquia $(r=0,50)$ e negativamente com os fatores Apoio $(r=-0,31)$ e Coesão $(r=-0,42)$. Já o fator Hierarquia apresentou correlações negativas com o fator Apoio $(r=-0,16)$ e coesão $(r=-0,16)$ e com o fator Coesão correlacionou-se positivamente com o fator Apoio $(r=0,53)$ (Teodoro et al., 2009).

Recentemente, um novo estudo desenvolvido por Matias e Teodoro (2018) investigou evidências de validade do Inventário do Clima Familiar para Crianças (ICF-C), na versão de autorrelato. O estudo foi conduzido em uma amostra de 183 crianças (57,4\% meninas), residentes em Belo Horizonte (MG), com idades entre 8 e 12 anos $(M=10,41$ anos; $D P=0,80)$. Os itens do ICF foram reescritos e simplificados para atender ao perfil do público-alvo (crianças) seguindo a proposta de Borsa, Damásio e Bandeira (2012). Os resultados indicaram que a estrutura da versão original da ICF não se confirmou na versão da ICF-C sendo que nesta última, os itens que se dividiram em dois fatores, denominados Clima Familiar Negativo (oito itens) e Clima Familiar Positivo (10 itens). As cargas fatoriais dos itens variaram entre 0,40 a 0,91 no primeiro fator $(\alpha=0,77)$ e entre 0,32 a 0,72 no segundo fator $(\alpha=0,67)$. 
É válido pontuar que pesquisas realizadas com o público infantil podem demandar a inclusão de novos agentes como informantes, em especial quando investiga-se características do desenvolvimento infantil e sua associação com aspectos contextuais, tais como a escola e a família (Borsa \& Muniz, 2016). A prática de incluir outros agentes como informantes, é, inclusive, imprescindível no contexto da avaliação psicológica infantil (Borsa \& Muniz, 2016; Pires, 2017). No contexto da investigação com crianças, instrumentos de autorrelato apresentam o viés da desejabilidade social, na medida em que as respostas tendem a representar o julgamento da criança diante do que é mais adequado socialmente, e não em seu próprio comportamento (Borsa \& Muniz, 2016). Esse viés é uma questão a ser observada por pesquisadores, especialmente quando procura-se avaliar tópicos sensíveis, como comportamentos de risco e comportamento alimentar (Miller et al., 2015). Instrumentos de heterorrelato, entretanto, também são passíveis de vieses. Geralmente adultos cuidadores ou professores são convidados a responder o instrumento e, assim, pode haver o receio do julgamento de um terceiro. Além disso, adultos têm a tendência de avaliar os comportamentos da criança a partir de seus próprios valores ou julgamentos, impedindo uma avaliação isenta e imparcial (Borsa \& Muniz, 2016).

Para minimizar determinado viés, é interessante recorrer a diferentes informantes e utilizar inúmeros métodos e técnicas (Borsa \& Muniz, 2016; Pritchett et al., 2011). Na avaliação de características associadas ao funcionamento familiar, estudo de revisão sistemática sobre instrumentos disponíveis na língua inglesa, concluiu que a maior parte dos instrumentos disponíveis exige apenas o input de um membro da família. Os autores sugerem a estudos futuros a combinação de medidas que recorram a múltiplos informantes, como uma forma de construir, por exemplo, medidas mais holísticas (Pritchett et al., 2011).

Dessa forma, levando em consideração a relevância do clima familiar para o desenvolvimento e saúde mental dos membros, (Ackerman et al., 2013; Leusin et al., 2018; Luebbe \& Bell, 2014) a importância da disponibilidade de instrumentos validados para público infantil, e levando em conta que o ICF destaca-se como a única escala, com evidências de validade no Brasil, para a avaliação do clima familiar (Teodoro et al., 2009), o objetivo do presente estudo é identificar a estrutura fatorial do ICF quando a forma de aplicação é heterorrelato. Em particular, quando o agente informante é o cuidador da criança, e não ela própria. A validação do instrumento em uma versão de heterorrelato permitirá não apenas a disponibilidade de mais uma ferramenta investigativa que integre a percepção de cuidadores, como também tornará possível, futuramente, estudos que analisem diferenças entre o reportado pelos cuidadores e pelas crianças em relação ao clima familiar.

\section{Método}

\section{Participantes}

Participaram do estudo 722 cuidadores de crianças, a média de idade foi $\left(M_{\text {idade }}=39,11 ; D P=7,38\right)$, sendo que $76,3 \%$ eram do sexo feminino $(n=551)$ e $14,1 \%$ do sexo masculino $(n=102)$ e um total de $9,6 \%(n=69)$ dos respondentes não informaram o sexo. Quanto à região de moradia, a amostra foi composta, principalmente, por residentes do Rio de Janeiro $(n=470)$, Rio Grande do Sul $(n=119)$, São Paulo $(n=57)$, Minas Gerais $(n=15)$, Paraná $(n=12)$, Paraíba $(n=8)$, Santa Catarina $(n=8)$, Distrito Federal $(n=6)$, Ceará $(n=5)$, dentre outros estados. Em relação às características das crianças pelas quais os cuidadores eram responsáveis, $47,5 \%$ eram meninos $(n=343)$ e $52,4 \%(n=378)$ meninas, e 1 caso não informou o sexo da criança $\left(M_{\text {idade }}=9,17 ; D P=1,59\right)$.

\section{Instrumentos}

Para a coleta de dados, foi utilizada do Inventário de Clima Familiar (ICF), descrito anteriormente, desenvolvido por Teodoro et al. (2009). Consiste em uma escala Likert, composto por 22 itens cujas respostas podem variam de 1 (Não concordo de jeito nenhum) a 5 (Concordo completamente). No estudo de construção e validação, a ICF apresentou estrutura composta por quatro fatores: "Coesão" $(\alpha=0,82$; e.g., "As pessoas gostam umas das outras"), "Apoio" $(\alpha=0,71$; e.g., "Ajudamos financeiramente uns aos outros"), "Hierarquia" $(\alpha=0,71$; e.g., "Os filhos têm pouco poder nas decisões familiares") e "Conflito" ( $\alpha=0,84$; e.g., "Briga-se por qualquer coisa"). Além do ICF, foi utilizado um questionário sociodemográfico, composto por perguntas fechadas para conhecer a idade, localidade e sexo dos participantes.

\section{Procedimentos de coleta de dados}

A amostra total do presente estudo foi constituída de duas subamostras, coletadas em dois estudos distintos, entre os anos de 2015 e 2017. Os dados de 268 participantes foram coletados de forma virtual, via plataforma on-line e os participantes foram recrutados pelas redes sociais por meio da técnica snowball (Patton, 1990). Já os dados de 554 participantes foram coletados presencialmente em escolas do Rio de Janeiro, por meio do envio do protocolo de instrumentos de pesquisa para os cuidadores responsáveis. Todos os participantes assinaram o Termo de Consentimento Livre e Esclarecido (TCLE), em suas versões on-line e impressa. As pesquisas que derivaram as duas amostras - virtual e presencial, foram submetidas e aprovadas pelo Comitê de Ética do Instituto de Psicologia da Universidade Federal do Rio Grande do Sul (CAAE: 05118412.6.0000.5334) e da Universidade Estadual do Rio de Janeiro (CAEE: 24367113.0.0000.5282), respectivamente. 


\section{Procedimentos de análise dos dados}

Para a avaliação de evidência de validade com base na estrutura interna do instrumento, realizou-se uma análise fatorial exploratória (EFA) no programa Factor Version 9.3 (Lorenzo-Seva \& Ferrando, 2006), com a amostra total de participantes $(n=722)$. A análise foi conduzida a partir de matriz de correlação policórica, tendo em vista o nível de medida ordinal característico do instrumento e da violação da hipótese e da normalidade multivariada dos dados (Holgado-Tello, Chacón-Moscoso, BarberoGarcia, \& Vila-Abad, 2010). Para tanto, utilizou-se o método de extração Robust Unweighted Least Squares (RULS, esse método pode ser utilizado em dados com distribuição normal e não normal e apresenta boa performance em bancos de dados com muitos itens (Damásio \& Dutra, 2018). Para a retenção de fator foi utilizado o método de análises paralelas (Optimal implementation of Parallel Analysis - PA), que é considerado um método apropriado na literatura (Lorenzo-Seva, Timmerman, \& Kiers, 2011). A confiabilidade da escala foi avaliada utilizando a fórmula de fidedignidade composta (Raykov, 1997) na ferramenta de calculadora online The Composite Reliability Calculator (Colwell, 2016).

\section{Resultados}

A partir da AFE, o método PA sugeriu dois fatores como a solução mais representativa dos dados (Kaiser-Meyer-Olkin [KMO] $=0.90049 ;$ Bartlett's test of sphericity $=5850,8 ; d f=231 ; p<0,001 ; \quad R M S E A=0,058$; $\mathrm{NNFI}=0,971$; WRMR $=0,0537$ ). $\mathrm{O}$ primeiro fator, aqui nomeado Clima Familiar Positivo, reuniu 10 itens cujo conteúdo representam comportamentos relacionados à coesão e apoio entre os membros da família. Já o segundo fator, denominado Clima Familiar Negativo, reuniu 12 itens associados à presença de conflito e autoritarismo entre os membros da família. Utilizando como ponto de corte cargas fatoriais maiores que 0,30 , todos os itens carregaram satisfatoriamente em um dos dois fatores, exceto o item 16 ("Os mais velhos mandam mais"), que apresentou carga fatorial cruzada de 0,437 e 0,389, respectivamente nos polos positivo e negativo do clima familiar. A solução de dois fatores foi responsável por $61,83 \%$ da variância total explicada (Tabela 1). Os fatores apresentaram correlação negativa $(r=-0,380 ; p<0,05)$. A fidedignidade composta foi de 0,895 para o fator Clima Familiar Positivo com o item 16 e 0,899 sem o item 16. Para o fator Clima Familiar Negativo foi 0,883 com o item 16 e $0,882 \mathrm{sem}$ o item 16. A estrutura fatorial manteve-se a mesma em ambas as configurações. As cargas fatoriais dos itens e a estrutura fatorial estão descritas na Tabela 1.

Tabela 1

Cargas Fatoriais da Versão de Heterorrelato do Inventário de Clima Familiar para Crianças

\begin{tabular}{|c|c|c|}
\hline \multirow[b]{2}{*}{ Item } & \multicolumn{2}{|c|}{ Cargas Fatoriais } \\
\hline & $\begin{array}{l}\text { Clima Familiar } \\
\text { Positivo }\end{array}$ & $\begin{array}{c}\text { Clima Familiar } \\
\text { Negativo }\end{array}$ \\
\hline $\begin{array}{l}\text { 1. Procuramos ajudar as pessoas da nossa família quando percebemos que } \\
\text { estão com problemas. }\end{array}$ & $0,536^{* *}$ & $-0,038$ \\
\hline 2. As proibições são constantes. & 0,130 & $0,407^{* *}$ \\
\hline 3. Uns mandam e outros obedecem. & 0,231 & $0,492^{* *}$ \\
\hline 4. As pessoas zombam umas das outras. & $-0,093$ & $0,643^{* *}$ \\
\hline 5. Briga-se por qualquer coisa. & $-0,011$ & $0,831^{* *}$ \\
\hline $\begin{array}{l}\text { 6. 6. Algumas pessoas deixam de fazer as suas coisas para auxiliar as outras } \\
\text { pessoas da família. }\end{array}$ & $0,417^{* *}$ & 0,144 \\
\hline $\begin{array}{l}\text { 7. 7. Não importa a vontade da maioria, a decisão final é sempre da mesma } \\
\text { pessoa. }\end{array}$ & $-0,091$ & $0,576^{* *}$ \\
\hline 8. As pessoas irritam umas às outras. & $-0,043$ & $0,755^{* *}$ \\
\hline 9. As pessoas gostam de passear e de fazer coisas juntas. & $0,668^{* *}$ & $-0,112$ \\
\hline 10. As pessoas resolvem os problemas brigando. & $-0,037$ & $0,788^{* *}$ \\
\hline 11. As pessoas criticam umas às outras frequentemente. & $-0,102$ & $0,742^{* *}$ \\
\hline 12. Resolver problemas significa discussão e brigas. & $-0,100$ & $0,680^{* *}$ \\
\hline 13. As pessoas tentam ajudar umas às outras quando as coisas não vão bem. & $0,750^{* *}$ & $-0,045$ \\
\hline 14. As pessoas gostam umas das outras. & $0,849^{* *}$ & $-0,002$ \\
\hline 15. Sinto que existe união entre os membros. & $0,816^{* *}$ & $-0,048$ \\
\hline 16. Os mais velhos mandam mais. & 0.437 & 0.389 \\
\hline 17. As pessoas se sentem próximas umas das outras. & $0,819^{* *}$ & $-0,057$ \\
\hline
\end{tabular}


Tabela 1 (continuação)

Cargas Fatoriais da Versão de Heterorrelato do Inventário de Clima Familiar para Crianças

\begin{tabular}{lcc}
\hline \multirow{2}{c}{ Item } & \multicolumn{2}{c}{ Cargas Fatoriais } \\
\cline { 2 - 3 } & $\begin{array}{c}\text { Clima Familiar } \\
\text { Positivo }\end{array}$ & $\begin{array}{c}\text { Clima Familiar } \\
\text { Negativo }\end{array}$ \\
\hline 18. O(s) filho(s) têm pouco poder nas decisões familiares. & 0,150 & $0,360^{* *}$ \\
19. Temos prazer e alegria em passar o tempo juntos. & $0,749^{* *}$ & $-0,124$ \\
20. Algumas pessoas resolvem os problemas de maneira autoritária. & $-0,017$ & $0,649^{* *}$ \\
21. Ajudamos financeiramente uns aos outros. & $0,645^{* *}$ & 0,086 \\
22. As pessoas me ajudam a fazer as coisas quando não tenho tempo. & $0,542^{* *}$ & 0,006 \\
Fidedignidade Composta & $0,899^{*}$ & $0,882^{*}$ \\
Variância explicada & 42.462 & 19.368 \\
\hline
\end{tabular}

Nota. *itens que carregaram satisfatoriamente nos fatores (carga fatorial $\geq 0,30$ ); Valores sem asterisco: itens que não carregaram (carga fatorial $\leq 0,30$ )

\section{Discussão}

O objetivo do presente estudo foi investigar evidências de validade da versão de heterorrelato do ICF. Os resultados da presente pesquisa indicaram bons índices psicométricos (Damásio \& Dutra, 2018; Pasquali, 2011; Yu \& Muthen, 2002) para a ICF - versão heterorrelato, apontando evidências iniciais de validade do instrumento, especificamente no que se refere à sua estrutura interna. A versão de heterorrelato do ICF, apresenta-se, então, potencialmente, como uma ferramenta disponível para a investigação do clima familiar a partir da perspectiva dos cuidadores. Esses resultados agregam uma nova ferramenta à prática de pesquisa brasileira, carente em instrumentos de mensuração de características da família (Matias \& Teodoro, 2017). Ainda, permitem a investigação de variáveis associadas ao desenvolvimento infantil a partir da perspectiva de outros informantes, complementando os dados obtidos por meio das medidas de autorrelato. Trata-se de uma estratégia importante para se conduzir um bom processo de investigação (De Los Reyes \& Kazdin, 2005).

O estudo original da ICF (Teodoro et al., 2009) encontrou uma solução de quatro fatores, denominados "Conflito", "Hierarquia", "Apoio" e "Coesão". No presente estudo, no entanto, encontrou-se uma solução de dois fatores, em que os itens referentes à presença de conflito (e.g., "Briga-se por qualquer coisa") e à presença de autoritarismo (e.g., "Não importa a vontade da maioria, a decisão final é sempre da mesma pessoa"), agruparam-se em um mesmo fator, indicando características relacionadas ao clima familiar negativo.

Itens referentes à presença de apoio (e.g., "Ajudamos financeiramente uns aos outros) e coesão (e.g., "Sinto que existe união entre os membros") agruparam-se em outro fator, indicando características relacionadas ao clima familiar positivo. A estrutura de dois fatores, aqui nomeadas "Clima Familiar Positivo" e "Clima Familiar
Negativo", está de acordo com a literatura, que identifica na complexidade do clima familiar características positivas, como o apoio oferecido na rede familiar e a manutenção da família como uma entidade coesa, e características negativas, tais como a presença de conflitos na família e uma gestão autoritária do sistema familiar (Björnberg \& Nicholson, 2007; Matias \& Teodoro, 2018; Teodoro et al., 2009).

A correlação negativa entre os fatores Clima Familiar Positivo e Clima Familiar Negativo ( $r=-0,380$; $p<0,05)$ também está em consistência com a versão original do instrumento, na qual se encontrou correlação positiva entre "Apoio" e "Coesão" (polo positivo) e entre "Hierarquia" e "Conflito" (polo negativo), além de correlação negativa entre ambos os fatores (Teodoro et al., 2009). A solução de dois fatores encontrada no presente estudo foi distinta daquela encontrada no estudo de construção e desenvolvimento da ICF (versão para adolescentes, Teodoro \& Allgayer, 2009) e semelhante àquela encontrada no estudo de adaptação da versão de autorrelato para crianças, a ICF-C (Matias \& Teodoro, 2018). Tais diferenças podem estar associadas ao método utilizado para a extração e retenção dos fatores (Damásio \& Dutra, 2018; Damásio, 2012; Howard, 2015). No estudo de Teodoro et al. (2009) realizou-se, inicialmente, uma análise fatorial exploratória utilizando como método de retenção de fatores o método dos eixos principais e análise do gráfico de sedimentação para auxiliar na decisão do número de fatores. Já no estudo de adaptação e validação do ICF-C, também foram utilizadas análises fatoriais exploratórias a partir do estimador Unweighted Least Squares (ULS) por meio de uma matriz de correlação policórica e análises paralelas para a retenção dos fatores (Matias \& Teodoro, 2018), o que pode explicar parcialmente a semelhança na solução fatorial encontrada. No presente estudo, realizou-se procedimentos semelhantes, como as análises paralelas (Optimal Implementation of Parallel Analysis) como procedimento para determinar o número 
de dimensões (Timmerman \& Lorenzo-Seva, 2011), e Robust Unweighted Least Squares (RULS) como método de extração de fatores e matriz de dispersão fundamentada em correlação policórica.

Outro achado do presente estudo que demanda discussão é o fato de o item 16 ("Os mais velhos mandam mais") ter carregado tanto no fator "Clima Familiar Positivo" quanto no fator "Clima Familiar Negativo". Tal resultado levou à decisão pela exclusão do item na estrutura final da versão de heterorrelato do ICF para crianças. $\mathrm{Na}$ versão de autorrelato originalmente desenvolvida e validada para adolescentes, o referido item carregou no fator denominado "Hierarquia", indicando que as ordens recebidas pelos pais e responsáveis são percebidas de forma negativa. Essa visão pode ser pensada a partir da compreensão de que na adolescência há um questionamento da autoridade e reestruturação de papéis apreendidos na infância (Stengel, 2011). Tal discrepância pode ser exemplificada pelo próprio resultado da versão da ICF-C (Matias \& Teodoro, 2018) em que o item "Os mais velhos mandam mais" carregou no fator positivo do Clima Familiar. Os autores discutem que essa visão dos mais velhos como figuras de autoridade é importante no desenvolvimento da moralidade infantil, justificando a pertinência de sua adesão ao fator positivo (Matias \& Teodoro, 2018). No presente estudo, o referido item apresentou maior carga no fator Clima Familiar Positivo $(0,437)$ quando comparada ao fator Clima Familiar Negativo $(0,389)$, sugerindo que o cuidador pode entender que a hierarquia é algo natural na relação entre pais e filhos.

Cabe ressaltar que, na caracterização tipológica sobre o estilo de controle parental, Baumrind (1966) propõe um modelo amplamente utilizado na literatura (Pinto et al., 2014), que são os três protótipos: autoritativo, autoritário e permissivo. O estilo autoritativo busca direcionar e orientar as atividades da criança, incentiva o diálogo, compartilha com a criança o raciocínio por trás de determinada ação, reconhecendo os interesses e particularidades da criança, não baseando a decisão em um consenso ou no desejo da criança (Baumrind, 1966; Weber, Prado, Viezzer, \& Brandenburg, 2004). O estilo autoritário, por outro lado, controla o comportamento dos filhos segundo regras estabelecidas e absolutas, valorizando a obediência e utilizando a punição como uma estratégia para lidar com o comportamento da criança (Baumrind, 1966; Weber et al., 2004). Por fim, o estilo permissivo evita estratégias punitivas e é receptivo diante dos desejos e ações da criança, apresentando-se como um meio de realizar os desejos e vontades infantis, porém sem empregar práticas responsáveis pelo processo de modelação ou orientação do comportamento (Baumrind, 1966).

Diante da caracterização de diferentes estilos parentais e do reconhecimento da contribuição desse modelo teórico para a compreensão do clima familiar (Björnberg \& Nicholson, 2007), é possível aferir que uma hierarquia no contexto familiar, em que os mais velhos teriam mais autoridade e mandariam mais, não é necessariamente percebido de forma negativa ou positiva pelos membros da família, em especial pelos filhos ou pelos mais novos. Nesse sentido, os itens relacionados ao fator original Hierarquia, na versão do ICF para adolescentes (Teodoro et al., 2009), parece melhor definido pelo termo "Autoritarismo", já que se referem a um determinado padrão de relacionamento mais associado ao estilo autoritário proposto por Baumrind (1966). Por exemplo, proibições constantes ou pouca participação dos membros da família nas tomadas de decisões são aspectos percebidos por eles, de forma geral, como negativos e associados a desfechos negativos em termos de desenvolvimento e saúde mental (Baumrind, 1966; Luebbe \& Bell, 2014; Brophy-Herb et al., 2013; Leusin et al., 2018). Em síntese, a decisão por excluir o item 16 na versão de heterorrelato dada a carga cruzada do item associa-se a sua possibilidade de distinta interpretação, dependendo do respondente e do seu papel na dinâmica familiar. Entende-se que relações familiares hierárquicas, em que os pais exercem autoridade sobre os filhos não é, por si só, positiva ou negativa. O que vai definir a qualidade dessa autoridade é a forma com que uma série de outras características familiares se apresentam na dinâmica familiar.

\section{Considerações Finais}

O presente estudo teve por objetivo apresentar evidências iniciais de validade interna da versão de heterorrelato do ICF, destinada aos pais e responsáveis de crianças. Os resultados indicaram adequadas propriedades psicométricas quanto à evidência de validade baseada na estrutura interna e pertinência com a literatura, identificando a presença de dois fatores, sendo eles Clima Familiar Positivo, Clima Familiar Negativo. No que diz respeito às limitações do estudo, destaca-se o fato de que a maior parte da amostra é proveniente da região Sudeste, o que pode prejudicar a representatividade da amostra em relação à população brasileira. Outra limitação é o fato de nem todos os respondentes terem informado qual o seu próprio sexo ou qual o sexo da criança, o que pode ter prejudicado uma melhor caracterização da amostra. Entretanto, como o objetivo do estudo não foi investigar invariância da medida entre sexo do respondente ou da criança, optou-se por manter todos os participantes na amostra final. Além disso, não houve um controle em relação aos respondentes em termo de nível de escolaridade e renda, o que poderia ser um dado importante para a discussão dos resultados.

Os resultados aqui presentes sugerem a adequação do instrumento para uso no contexto brasileiro. No entanto, novos são necessários para acrescentar evidências de validade para a ICF (AERA; APA; NCME, 1999, p. 11). Especificamente, faz-se relevante investigar 
evidências de validade da ICF com base na relação com variáveis externas, especificamente com outras medidas que mensuram características familiares. Futuramente, estudos buscando a relação entre as versões de heterorrelato e autorrelato da ICF permitirão compreender em que medida o viés do informante pode interferir nos resultados obtidos, tema de pesquisa ainda pouco explorado no Brasil. Além disso, será possível realizar comparações entre os dados oriundos de múltiplos informantes sobre o clima familiar e na percepção de crianças. Além disso, sugerem-se estudos comparativos, investigando, por exemplo, diferenças nos dados entre respondentes por crianças do sexo masculino e feminino ou responsáveis por crianças de diferentes escolaridades, e estudos que visem contemplar uma parcela ampla da população brasileira com distribuição geográfica heterogênea.

\section{Referências}

Ackerman, R. A., Kashy, D. A., Donnellan, M. B., Neppl, T., Lorenz, F. O., \& Conger, R. D. (2013). The interpersonal legacy of a positive family climate in adolescence. Psychological Science, 24(3), 243-250. doi: 10.1177/0956797612447818

American Educational Research Association [AERA], the American Psychological Association [APA] and the National Council on Measurement in Education [NCME] (1999). Standards for educational and psychological testing. Washington, DC: American Educational Research Association.

Baumrind, D. (1966). Effects of authoritative control on child behavior. Child Development, 37(4), 887-907. doi: 10.2307/1126611

Björnberg, A., \& Nicholson, N. (2007) The family climate scales - Development of a new measure for use in family business research. Family Business Review, 20(3), 229-246. doi: 10.1111/j.1741-6248.2007.00098.x

Borsa, J. C., Damásio, B. F., Bandeira, D. R. (2012). Adaptação e validação de instrumentos psicológicos entre culturas: Algumas considerações. Paidéia, 22(53), 423-432. doi: 10.1590/S0103-863X2012000300014

Borsa, J. C., \& Muniz, M. (2016). Testagem Psicológica de Crianças e Adolescentes. Em C. S. Hutz, D. R. Bandeira, C. M. Trentini, J. S. Krug (Eds.). Psicodiagnóstico (vol. 1,1 1a ed, pp. 238-246), Porto Alegre: Artmed.

Bronfenbrenner, U. (1979/1996). A ecologia do desenvolvimento humano: Experimentos naturais e planejados. Porto Alegre: Artes Médicas.

Brophy-Herb, H. E., Martoccio, T. L., Hillaker, B., Stansbury, K. E., Harewood, T., Senehi, N., \& Fitzgerald, H. (2013). Profiles of lowincome maternal well-being and family climate: Relations to Toddler Boys' and Girls' Behaviors. Family Relations, 62(2), 326-340. doi: 10.1111/fare.12001

Colwell, S. R. (2016). The composite reliability calculator. Technical Report. doi: 10.13140/RG.2.1.4298.088

Damásio, B. F. (2012). Uso da análise fatorial exploratória em psicologia. Avaliação Psicológica, 11(2), 213-228. Recuperado de http://pepsic. bvsalud.org/pdf/avp/v11n2/v11n2a07.pdf

Damásio, B. F., Dutra, D. F. (2018). Análise fatorial exploratória: Um tutorial com o software Factor. Em B. F. Damásio \& J. C Borsa, (Eds.) Manual de desenvolvimento de instrumentos psicológicos (1 ${ }^{\mathrm{a}}$ ed., pp. 241-265), São Paulo: Vetor.

De Los Reyes, A., \& Kazdin, A. (2005). Informant discrepancies in the assessment of childhood psychopathology: A critical review, theoretical framework, and recommendations for further study. Psychological Bulletin, 131(4), pp. 483-509.

Howard, M. (2015) A review of exploratory factor analysis (EFA) decisions and overview of current practices: What we are doing and how can we improve? International Journal of Human-Computer Interaction, 32(1), 51-62. doi: 10.1080/10447318.2015.1087664

Holgado-Tello, F., Chacón-Moscoso, S., Barbero-García, I., \& Vila-Abad, E. (2010). Polychoric versus Pearson correlations in exploratory and confirmatory factor analysis of ordinal variables. Quality and Quantity, 44(1), 153-166. doi: 10.1007/s11135-008-9190-y

Kurdek, L. A., Fine, M. A., \& Sinclair, R. J. (1995). School adjustment in sixth graders: Parenting transitions, family climate, and peer norm effects. Child Development, 66(2), 430-445. doi: 10.2307/1131588

Leusin, J. F., Petrucci, G. W., \& Borsa, J. C. (2018). Clima Familiar e os problemas emocionais e comportamentais na infância. Revista da SPAGESP, 19(1), 49-61. Recuperado de http://pepsic.bvsalud.org/pdf/rspagesp/v19n1/v19n1a05.pdf

Lorenzo-Seva, U., \& Ferrando, P. J. (2006). FACTOR: A computer program to fit the exploratory factor analysis model. Behavioral Research Methods, Instruments and Computers, 38(1), 88-91. doi: 10.3758/BF03192753

Lorenzo-Seva, U., Timmerman, M. E., \& Kiers, H. A. L. (2011). The Hull Method for selecting the number of common factors. Multivariate Behavioral Research, 46(2), 340-364. doi: 10.1080/00273171.2011.564527

Luebbe, A. M., \& Bell, D. J. (2014). Positive and negative family emotional climate differentially predict youth anxiety and depression via distinct affective pathways. Journal of Abnormal Child Psychology, 42(6), 897-911. doi: 10.1007/s10802-013-9838-5

Matias N. C. F., \& Teodoro M. L. M. (2018). Adaptação do inventário do clima familiar para crianças (ICF-C). Avaliação Psicológica 17(1), 20-27. doi: 10.15689/ap.2017.1701.03.13059

Matos, M. B., Cruz, A. C., Dumith, S. C., Dias, N. C., Carret, R. B., \& Quevedo, L. A. (2014). Eventos estressantes na família e indicadores de problemas de saúde mental em crianças com idade escolar. Ciência E Saúde Coletiva, 20(7), 2157-2163. doi:10.1590/141381232015207.17452014

Marturano, E. M., \& Elias, L. C. S. (2016). Família, dificuldades no aprendizado e problemas de comportamento em escolares. Educar em Revista, (59), 123-139. doi: 10.1590/0104-4060.44617

Miller, P. H., Baxter, S. D., Royer, J. A., Hitchcock, D. B., Smith, A. F., Collins, K. L., ... Finney, C. J. (2015). Children's Social Desirability: Effects of Test Assessment Mode. Personality and individual differences, 83, 85-90. doi: 10.1016/j.paid.2015.03.039

Minuchin, S. (1995). A cura da família: Histórias de esperança e renovação contadas pela terapia familiar. Porto Alegre: Artes Médicas.

Moos, R. H., \& Moos, B. S. (1994). Family environment scale. Manual. (3 ed.) Palo Alto, CA: Consulting Psychologists Press.

Olson, D. H., Portner, J., \& Lavee, Y. (1985). Faces III. St. Paul, MN: University of Minnesota, Family Social Science.

Pasquali, L. (2011). Psicometria: teoria dos testes na Psicologia e Educação (4⿳a ed.). Editora Vozes: Petrópolis. 
Patton, M. (1990). Qualitative evaluation and research methods. Beverly Hills, CA: Sage.

Phillips, T. M. (2012) The influence of family structure vs. family climate on adolescent well-being. Child and Adolescent Social Work Journal, 29(2), 103-110. doi: 10.1007/s10560-012-0254-4

Pinto, H. M., Carvalho, A. R., \& Sá, E. N. (2014). Os estilos educativos parentais e a regulação emocional: Estratégias de regulação e elaboração emocional das crianças em idade escolar. Análise Psicológica, 32(4), 387-400. doi: 1014417/ap.32.3.844

Pires, E. U. (2017). Avaliação de crianças e adolescentes: Aspectos cognitivos. Em M. R. C. Lins \& J. C. Borsa (Eds.). Avaliação Psicológica: Aspectos teóricos e práticos (vol. 1,1 $1^{\mathrm{a}}$ ed, pp. 213-235), Petrópolis, RJ: Vozes.

Pritchett, R., Kemp, J., Wilson, P., Minnis, H., Bryce, G., \& Gillberg, C. (2011). Quick, simple measures of family relationships for use in clinical practice and research. A systematic review. Family Practice, 28(2), 172-187. doi: 10.1093/fampra/cmq080

Raykov, T. (1997). Estimation of composite reliability for congeneric measures. Applied Psychological Measurement, 21(2), 173-184. doi: $10.1177 / 01466216970212006$

Stengel, M. (2011). O exercício da autoridade em famílias com filhos adolescentes. Psicologia em Revista, 17(3), 502-521. Recuperado de http://pepsic.bvsalud.org/pdf/per/v17n3/v17n3a11.pdf

Teodoro, M. L. M., Allgayer, M., \& Land, B. (2009). Desenvolvimento e validade fatorial do Inventário do Clima Familiar (ICF) para adolescentes. Psicologia: Teoria e Prática, 11(3), 27-39. Recuperado de http://pepsic.bvsalud.org/pdf/ptp/v11n3/v11n3a04.pdf

Timmerman, M. E., \& Lorenzo-Seva, U. (2011). Dimensionality Assessment of Ordered Polytomous Items with Parallel Analysis. Psychological Methods, 16(2), 209-220. doi: 10.1037/a0023353.

Valdés Cuervo, Á. A., \& Carlos Martínez, E. A. (2014). Relación entre el autoconcepto social, el clima familiar y el clima escolar con el bullying en estudiantes de secundaria. Avances en Psicología Latinoamericana, 32(3), 447-457. doi: 10.12804/apl32.03.2014.07

Weber, L. N. D., Prado, P. M., Viezzer, A. P., \& Brandenburg, O. J. (2004). Identificação de estilos parentais: O ponto de vista dos pais e dos filhos. Psicologia: Reflexão e Crítica, 17(3), 323-331. doi: 10.1590/S0102-79722004000300005

Yu, C., \& Muthen, B. (2002). Evaluation of model fit indices for latent variable models with categorical and continuous outcomes. Paper presented at the annual meeting of the American Educational Research Association, New Orleans, L.A.

\section{Sobre as autoras}

Laila Pires Ferreira Akerman é Psicóloga (Universidade Federal do Rio de Janeiro - UFRJ), mestranda em Psicologia Clínica na Pontifícia Universidade Católica do Rio de Janeiro (PUC-Rio). Bolsista CNPq.

Juliane Callegaro Borsa é Adjunta do Departamento de Psicologia e do Programa de Pós-Graduação em Psicologia Clínica da Pontifícia Universidade Católica do Rio de Janeiro (PUC-Rio), Coordenadora do Grupo de Pesquisa em Avaliação Psicológica APlab - Pessoas \& Contextos. Bolsista Produtividade CNPq Nível 2 (2017-) e Bolsista Jovem Cientista do Nosso Estado FAPERJ (2016-). É psicóloga, com mestrado em Psicologia Clínica pela Pontifícia Universidade Católica do Rio Grande do Sul (PUCRS), Doutorado e estágio de Pós-Doutorado em Psicologia pela Universidade Federal do Rio Grande do Sul (UFRGS). 